\title{
Optical memory: introduction by the feature editors
}

\author{
Mark A. Neifeld, Demetri Psaltis, and Lambertos Hesselink
}

The information age has brought with it an everincreasing demand for storage systems that have high capacity, fast data-transfer rate, and short access time. Vast memory systems are required for supporting the many storage-intensive computer applications such as multimedia, video on demand, and network-based computing. Optical memories already play an important role in existing commercial systems. The current role of optical disk memory (magneto-optical and CD-ROM) is driven primarily by its convenient removability and ease of replication. Progress on such planar storage systems continues. The recent demonstration of multilayer optical-disk-based storage has renewed research interest in this format, which now promises to offer significant capacity increases with minimal modification to the supporting interface (i.e., optical and electronic) systems.

The natural extension of discrete multilayer bitwise storage has also seen significant progress with demonstrations of bit-wise volume storage using photopolymers, two-photon materials, and photorefractives. An alternative to volume bit-wise storage is volume holographic storage. Recent advances in materials, spatial-light-modulator technologies, and CCD technologies have made possible demonstrations of volume holographic optical memories that offer the desired combination of high capacity, fast transfer rate (by means of parallel access), and short

M. A. Neifeld is with the Department of Electrical and Computer Engineering, University of Arizona, 1230 East Speedway, Tucson, Arizona 85721. D. Psaltis is with the Department of Electrical Engineering, California Institute of Technology, MS 116-81, 1201 E. California Boulevard, Pasadena, California 91125. L. Hesselink is with the Department of Electrical Engineering, Stanford University, MC 4035 353, Durand Building, Stanford, California 94305.

Received 22 January 1996.

0003-6935/96/142345-01\$10.00/0

(1) 1996 Optical Society of America retrieval time (by means of nonmechanical scanning). In addition to angular and wavelength multiplexing, other novel multiplexing techniques have emerged, and combinations of these are now routinely used to achieve high storage capacity. Many of these benchtop demonstration experiments have found their way into near-commercial-ready holographic storage systems, and issues related to cost, compactness, and signal fidelity have become a central focus of the research activity.

These latest system demonstrations have pointed to the need to study interface issues and noise in the context of parallel-access optical memory. Interface components such as page buffers, signal processors, and decoders have been presented for both general and special purpose (e.g., database) memory applications, while researchers continue to find novel methods for the use of parallel optical memory access within future electronic- and optical-computing platforms.

The contributions to this feature issue represent a wide range of topics in optical memory. The breadth of optical-memory activities represented by the above discussion is addressed well by the papers in this feature issue, and the near-term potential of volume optical storage techniques can be appreciated from the large number of system-demonstration papers contained herein. From improved quadrant pupil detection techniques for conventional optical-diskbased storage to three-dimensional holographic disks using novel multiplexing to sophisticated signal processing and detection techniques for page access to a compact holographic storage demonstrator, this feature issue is intended to bring together recent developments in order that the reader might appreciate the rapid progress in and the near-term potential of the many forms of optical memory.

Finally, the guest editors acknowledge the many efforts of the reviewers and the Applied Optics staff in the preparation of this feature issue. 